\title{
COMBINED EFFECT OF BROMELAIN AND TURMERIC AGAINST ACETIC ACID INDUCED ULCERATIVE COLITIS IN WISTAR RATS
}

\author{
R. Padmavathi*, K. B. Keerthana, A. Lalitha Devi, Y. Sree Hari, Abdul Samad
}

\section{Article Information}

Received: $19^{\text {th }}$ September 2020

Revised: 24 ${ }^{\text {th }}$ March 2021

Accepted: $12^{\text {th }}$ April 2021

Keywords

Disease activity index, macroscopic score, bromelain, myeloperoxidase, ulcerative colitis

\begin{abstract}
Objective: The present study planned to study the combined effect of Bromelain and Turmeric in acetic acid-induced ulcerative colitis in Wistar rats. Methodology: Wistar rats of either sex $(n=30)$ were divided into 5 groups. Group - I, Sham control, administered a single dose of vehicle rectally; Group II, colitis control, colitis was induced by rectal administration of a single dose of $2 \mathrm{ml}$ of 3\%, v/v of acetic acid in $0.9 \%$ saline; Group-III, treated with Turmeric (50 mg/kg/p.o); Group-IV, treated with Bromelain (100 mg/kg/p.o) and Group -V, treated with both Turmeric (50 mg/kg/p.o) and Bromelain (100 mg /kg/p.o). Colitis was induced in all the treatment groups on the first day and drug treatment was continued for 7 days. During the treatment period, Disease Activity Index (DAI) was assessed daily and on the $8^{\text {th }}$ day White Blood Cell (WBC) Count and Differential Leucocyte Count (DLC) was performed and on the $9^{\text {th }}$ day, all the rats were sacrificed for the assessment of intestinal inflammation, colon myeloperoxidase (MPO) levels and, Histopathology. Results obtained were analyzed by one-way analysis of variance followed by Tukey’s multiple comparison test. Results: Overall changes in DAI, Inflammatory scores, WBC, DLC and, MPO have shown significant improvement with Turmeric and Bromelain compared to Colitis control. However, the combined therapy has shown to be more effective in alleviating ulcerative colitis compared to individual therapies. Conclusion: The present study recommends that the combination of Turmeric and Bromelain has a synergistic effect in treating ulcerative colitis.
\end{abstract}

\section{INTRODUCTION}

Inflammatory bowel diseases (IBDs) are chronic inflammatory disorders of the gastrointestinal tract. The term IBD describes two specific diseases: ulcerative colitis (UC) and Crohn's disease (CD) [1]. These disorders were first described by Sir Samuel Wilks and Doctor Crohn [2,3]. Ulcerative colitis is characterized by inflammation that starts in the rectum, spreads proximally to other regions of the colon and, sometimes involves

*G. Pulla Reddy College of Pharmacy, Hyderabad - 500028 - Telangana - India

\section{*For Correspondence: rpvathi79@gmail.com}

This is an Open Access article distributed under the terms of the Creative Commons Attribution (CC BY NC), which permits unrestricted use, distribution, and reproduction in any medium, as long as the original authors and source are cited. No permission is required from the authors or the publishers. (https://creativecommons.org/licenses/by-nc/4.0/) 
the peri-appendiceal region. Whereas, Crohn's disease is characterized by inflammation, which is non-continuous and spreading across the gastrointestinal tract, most commonly the terminal ileum or the perianal region. It is commonly associated with complications such as strictures, abscesses and, fistulas [4].

The annual incidence of ulcerative colitis and Crohn's disease ranges from 1 to 10 cases per 100,000 people across different regions [5]. The incidence and prevalence of IBD are found to be high in industrialized nations in North America and Europe and have been increasing in recent years in previously lowincidence areas such as Asia [6].

Although the exact etiology of the pathogenesis of ulcerative colitis is largely unknown, growing evidence specifies the involvement of multiple etiological factors such as an individual's genetic susceptibility, external environment, intestinal microbial flora and, immune responses [7]. Though conventional treatments are effective in maintaining remission and decreasing the length of active disease periods, unfortunately, the long-term usages of these agents are reported to be associated with undesirable side effects and compromise the therapeutic advantage. Therefore, there is a need for the investigation of novel agents that are effective and safe for humans. Turmeric, a dry rhizome of Curcuma longa Linn (Zingiberaceae), has been used for centuries in indigenous medicine. Some of the recent studies revealed that curcumin, an active component of turmeric was found to be beneficial in preventing or ameliorating UC and inflammation [8]. The major problem associated with curcumin is its poor bioavailability due to its rapid metabolism in the liver and intestinal wall. Curcumin, therefore, could be a therapeutic option for the treatment of different diseases, provided limitations in its oral bioavailability can be overcome [9]. A Significant number of studies proved that adding bioavailability enhancer to curcumin enhanced its clinical effectiveness [10]. Studies are reporting the beneficial effect of bromelain in inflammatory conditions and also as a bioavailability enhancer [11,12].

Till now, no study has been done to investigate the effect of the combination of Turmeric and Bromelain in ulcerative colitis. Therefore, the present study was planned to investigate the combined effect of Bromelain and Turmeric in acetic acidinduced ulcerative colitis using Wistar rats. The potential of treatments was evaluated by assessing disease activity index
(DAI), ulcer index, WBC and DLC, myeloperoxidase (MPO), and histopathological studies.

\section{MATERIALS AND METHODS Chemicals}

WBC fluid and Agar-agar were purchased from S.D. Fine chemical Ltd. Leishman's stain

was purchased from NICE-Chemicals Pvt Ltd. Hexadecyl trimethyl ammonium bromide and Orthodianisidine hydrochloride were purchased from Sigma Aldrich. Bromelain, Turmeric and, rectal catheters were purchased from local stores.

\section{Experimental Animals}

Wistar rats of either sex (150-250 g) were obtained from National Centre for Laboratory Animal Sciences, National Institute of Nutrition, Hyderabad, India. They were maintained under standard laboratory conditions (12-h light/dark cycle, $25 \pm 3^{\circ} \mathrm{C}$, and $45-46 \%$ humidity) and provided with standard rat feed and water ad libitum. All the rats were acclimatized to standard laboratory conditions for one week before starting the experiment.

All the experimental procedures were done following the "Committee for the Purpose of Control and Supervision of Experiments on Animals” (320/CPCSCEA dated 03-01 2001) guidelines. The study was reviewed and approved by the Institutional Animal Ethics Committee (GPRCP/IAEC), G. Pulla Reddy College of Pharmacy, Hyderabad, India.

\section{Experimental protocol}

Rats were randomly allocated to five groups $(n=6)$. Group - I, Sham control administered a single dose of vehicle rectally; Group -II, colitis control, colitis was induced by rectal administration of a single dose of $2 \mathrm{ml}$ of 3\%, v/v of acetic acid in $0.9 \%$ saline; Group-III, treated with Turmeric (50 mg/kg/p.o); Group-IV, treated with Bromelain (100 mg/kg/p.o) and Group $-\mathrm{V}$, treated with both Turmeric (50 mg/kg/p.o) and Bromelain (100 mg /kg/p.o). Colitis was induced in all the treatment groups on the first day and drug treatment was continued for 7 days. During the treatment period Disease activity index (DAI) was assessed daily and on the 8th day the rats were anesthetized with Isoflurane and blood was collected by a retro-orbital puncture for WBC \& DLC Count. On the $9^{\text {th }}$ day animals were sacrificed by cervical dislocation (under Isoflurane anesthesia) and the colon was dissected out and flushed gently with saline and it was 
used for macroscopic scoring, MPO estimation, and Histopathology.

\section{Induction of colitis}

The animals were fasted overnight with free access to water. On the next day, they were anesthetized and a rectal catheter with 2 mm diameter was inserted through the rectum into the colon to a distance of $8 \mathrm{~cm}$. A solution of $2 \mathrm{ml}$ of acetic acid (3\%,v/v) in $0.9 \%$ saline was instilled into the lumen of the colon and maintained in a supine Trendelenburg position for $30 \mathrm{sec}$ to prevent leakage [13].

\section{Assessment of DAI score of colitis}

Colitis was quantified with the DAI scoring system by assessing weight loss, stool consistency, and rectal bleeding on daily basis for 7 days during the treatment period in all groups (Table 1) [13].

Table 1: Scoring for DAI

\begin{tabular}{llll}
\hline Score & $\begin{array}{l}\text { Weight } \\
\text { Loss (\%) }\end{array}$ & Stool Consistency & $\begin{array}{l}\text { Rectal } \\
\text { Bleeding }\end{array}$ \\
\hline 0 & $\begin{array}{l}\text { No weight } \\
\text { Loss }\end{array}$ & $\begin{array}{l}\text { Well-formed pellets } \\
\text { ho blood in } \\
\text { hem occult }\end{array}$ \\
\hline 1 & $1-5$ & $\begin{array}{l}\text { Pasty \& semi-formed } \\
\text { that didn't stick to the } \\
\text { anus }\end{array}$ & $\begin{array}{l}\text { Positive } \\
\text { hem occult }\end{array}$ \\
\hline 2 & $5-10$ & $\begin{array}{l}\text { Pasty \& semi-formed } \\
\text { that didn't stick to the } \\
\text { anus }\end{array}$ & $\begin{array}{l}\text { Positive } \\
\text { hem occult }\end{array}$ \\
\hline 3 & $10-20$ & $\begin{array}{l}\text { Pasty \& semi-formed } \\
\text { that didn't stick to the } \\
\text { anus }\end{array}$ & $\begin{array}{l}\text { Positive } \\
\text { hem occult }\end{array}$ \\
\hline 4 & Above 20 & $\begin{array}{l}\text { Liquid stools that did } \\
\text { stick to the anus }\end{array}$ & $\begin{array}{l}\text { Gross } \\
\text { bleeding }\end{array}$ \\
\hline
\end{tabular}

\section{Estimation of WBC and Neutrophils}

Blood was collected on the $8^{\text {th }}$ day through a retro-orbital puncture and collected into sample tubes coated with EDTA. Blood sample from the tube was drawn up to the 0.5 mark on the WBC pipette and, then mixed with WBC diluting fluid up to mark 11 and allowed for $10 \mathrm{~min}$ for then erythrocytes to lyse. Thereafter, the Neubauer counting chamber was filled with the diluted sample and WBC was counted microscopically under 40 $x$ magnification. The total numbers of white cells in the 4 large squares are counted and at the end, the total white cell count. WBC is calculated from the formula,

$$
W B C=n \times 20 \times 2.5
$$

where $n$ is the number of cells counted. The dilution factor is $1: 20$ and the volume factor for the counting chamber is $1 / 0.4$ (i.e. 2.5).

The Leishman staining technique was used for the estimation of neutrophil count. A drop of blood was placed on one end of the glass slide. The coverslip was used to make a blood smear on the glass slide using the push wedge technique. The Leishman was applied on the blood film and then left to stand for $2 \mathrm{~min}$. Thereafter, distilled water, two times the quantity of stain was used to flood the thin film of the blood; the setup was mixed gently for $2 \mathrm{~min}$ and then allowed to stand for 15 min before rinsing the stain. The slide was left to dry and counting was done on the microscope under oil immersion using 100x magnification [14].

\section{Assessment of macroscopic characters}

The animals were sacrificed and the colon was excised and opened longitudinally. Macroscopic scoring was done using the following score criteria, 0 - No change in both mucosa and serosa, 1- Petechial bleeding and hyperemia, 2-single mucosal erosion or ulceration, 3- single mucosal erosion or ulcer with hyperemic, adhesive, or hemorrhagic lesions in the serosa and mesentery, 4-multiple erosions or ulcerations (less than $10 \mathrm{~cm}$ ) and 5-Multiple erosions or ulcerations (more than $10 \mathrm{~cm}$ ) [15].

\section{Estimation of colonic MPO level}

The colon was taken, weighed (500mg) and, rinsed with ice-cold saline. Then it was blotted dry, weighed and, minced. The minced tissue was homogenized in 10 volumes of ice-cold potassium phosphate buffer ( $\mathrm{pH}$ 7.4) using a Remi tissue homogenizer. The homogenate was centrifuged at $3500 \mathrm{rpm}$ for $30 \mathrm{~min}$ at $4^{\circ} \mathrm{C}$. The supernatant was discarded.

The pellet was collected and $10 \mathrm{ml}$ of ice-cold $50 \mathrm{mM}$ potassium phosphates buffer ( $\mathrm{pH}$ 6.0) containing 0.5\% hexadecyl trimethyl ammonium bromide and $10 \mathrm{mM}$ ethylene diamine tetra-acetic acid was added. It was then subjected to one cycle of freezing and thawing and subjected to sonication for 15 seconds. The resulting solution was centrifuged at 15,000 rpm for $20 \mathrm{~min}$. From this $0.1 \mathrm{ml}$ of supernatant was taken added with $2.9 \mathrm{ml}$ of $50 \mathrm{mM}$ phosphate buffer containing $0.167 \mathrm{mg} / \mathrm{ml}$ O-dianisidine hydrochloride and $0.0005 \% \mathrm{H} 2 \mathrm{O} 2$. The change in absorbance was measured spectrophotometrically at $460 \mathrm{~nm}$. One unit of MPO activity is defined as the change in absorbance per minute 
by 1.0 at room temperature in the final reaction. MPO activity is calculated by

MPO activity $\left(\frac{U}{g}\right)=\frac{X}{\text { weight of the piece of tissue taken }}$

Where $\mathrm{X}=10 \mathrm{X}$ changes in absorbance per minute / Volume of supernatant taken in the final reaction [15].

\section{Histopathology}

A small segment of the colon was fixed in phosphate-buffered formaldehyde and embedded in paraffin. Tissue sections of $5 \mu \mathrm{m}$ were prepared, stained with hematoxylin and eosin and, were evaluated by light microscopy [15].

\section{Statistical analysis}

Results were expressed as Mean \pm S.E.M. The statistical significance was assessed using "One way Analysis of Variance” (ANOVA) followed by Tukey’s multiple comparison test using Graph pad prism 7.0 software. $\mathrm{P}<0.05$ was considered significant.

\section{RESULT AND DISCUSSION}

Effect of Bromelain and Turmeric on Disease Activity Index (DAI)

As shown in Figure 1, the DAI scores reflect the highest incidence of weight loss, diarrhea and, rectal bleeding in the colitis control group compared to the sham control group $(\mathrm{p}<$ 0.0001), whereas treatment with Turmeric $(\mathrm{p}<0.005)$ and Bromelain $(\mathrm{p}<0.01)$ alone has significant improvement in DAI scores as compared to the colitis control group. However, the combination of Bromelain and Turmeric has shown highly significant improvement in DAI scores, resuming to basal scores ( $\mathrm{p}<0.0001)$. Results are expressed as Mean \pm S.E.M , $\square \mathrm{P}<$ 0.0001 vs. Sham control. ${ }^{\mathrm{a}} \mathrm{P}<0.005$, ${ }^{\mathrm{b}} \mathrm{P}<0.01$, ${ }^{\mathrm{c}} \mathrm{P}<0.0001$ vs. Colitis control ${ }^{\#} \mathrm{P}<0.02$ vs. Combination.

\section{Disease activity index}

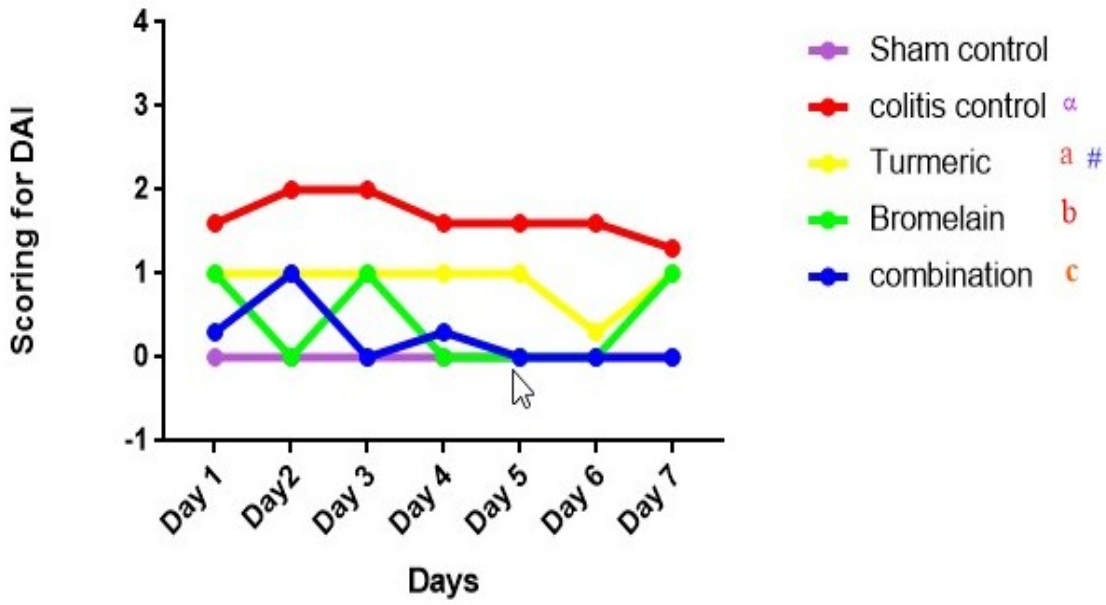

Figure 1: Effect of Bromelain and Turmeric on DAI

Effect of Bromelain and Turmeric on WBC and neutrophil count

Table 2: Effect of Bromelain and Turmeric on WBC and Neutrophil count.

\begin{tabular}{|l|l|l|}
\hline Groups $(\mathbf{n = 6 )}$ & $\begin{array}{l}\text { WBC Count } \\
\text { (cells } / \mathbf{~ m m 3})\end{array}$ & $\begin{array}{l}\text { Neutrophil count } \\
(\mathbf{\%})\end{array}$ \\
\hline Sham control & $6558 \pm 328.2$ & $57 \pm 1.528$ \\
\hline Colitis control & $12700 \pm 186.6^{\alpha}$ & $76.67 \pm 1.145^{\alpha}$ \\
\hline Turmeric & $8058 \pm 253.1^{\mathrm{a} \#}$ & $58.67 \pm 1.994^{\mathrm{a}}$ \\
\hline Bromelain & $7250 \pm 246.0^{\mathrm{a}}$ & $55.17 \pm 1.6^{\mathrm{a}}$ \\
\hline Combination & $6575 \pm 182.5^{\mathrm{a}}$ & $55.17 \pm 1.797^{\mathrm{a}}$ \\
\hline
\end{tabular}

Results are expressed as Mean \pm S.E.M , ${ }^{\alpha} \mathrm{P}<0.0001$ vs. Sham control. ${ }^{\text {a }} \mathrm{P}<0.0001$ vs. Colitis control. ${ }^{\#} \mathrm{P}<0.002$ vs. Combination.

Induction of colitis has significantly increased WBC count in the colitis control group compared to the sham control group $(\mathrm{P}<$ 0.0001). Treatment with turmeric and bromelain alone has shown a significant decrease in WBC count compared to the colitis control group $(\mathrm{P}<0.0001)$. A More significant decrease was shown upon treating with the combination of bromelain and turmeric $(\mathrm{P}<0.002)$ compared to individual treatments and also resumed to basal levels. Colitis induction significantly increased neutrophil count in the colitis control group compared to the sham control group $(\mathrm{P}<0.0001)$. Upon treatment with Turmeric 
and Bromelain alone has shown a significant decrease in neutrophils compared to the colitis control group ( $\mathrm{P}<0.0001)$, and there was no significant decrease in neutrophil count upon treating with the combination [Table 2].

\section{Effect of Bromelain and Turmeric on Macroscopic Scoring} Induction of colitis significantly increased the macroscopic score in the colitis control group compared to the sham control group ( $\mathrm{P}<0.0001)$. Treatment with Turmeric and Bromelain alone has shown a significant decrease in macroscopic scoring $(\mathrm{P}<0.0001)$ and treating with the combination of Turmeric and Bromelain has shown a significant reduction in the macroscopic score compared to monotherapies $(\mathrm{P}<0.002)$ [Figure 2 \& Figure 3].

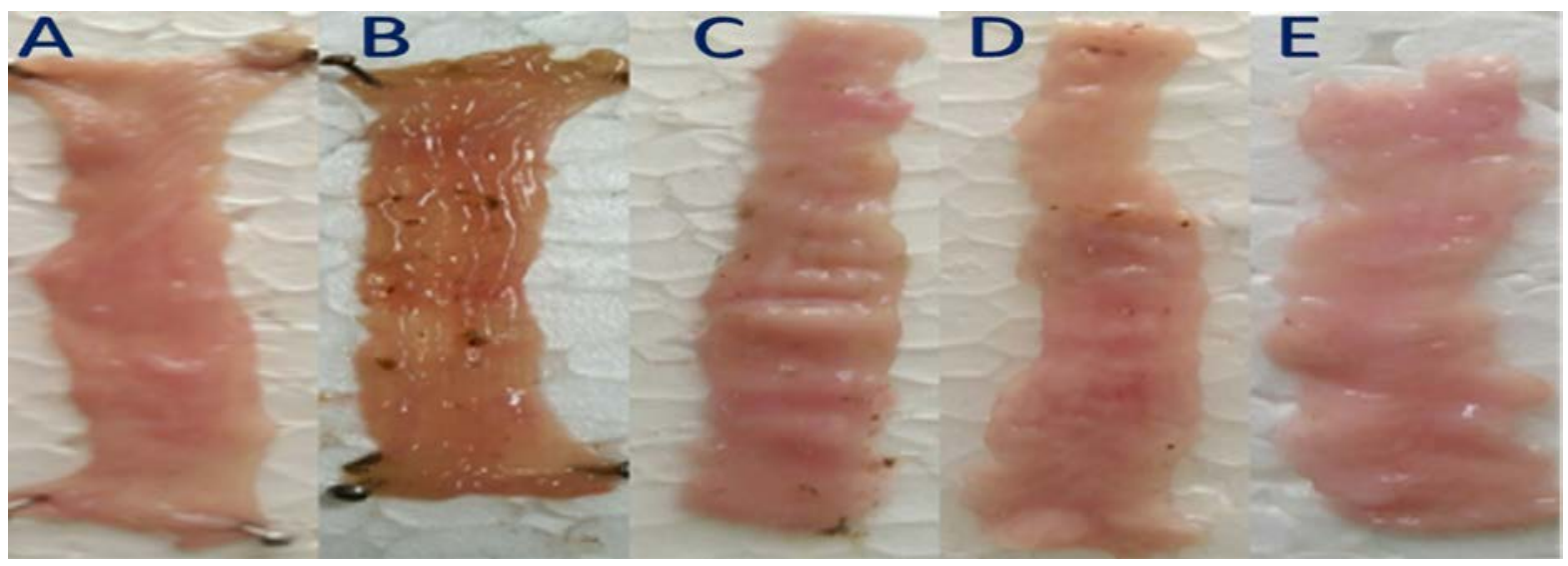

Figure 2: Macroscopic Scoring for Colon

Macroscopic presentation of acetic acid-induced ulcerative colitis in rats. A: Sham control, B: Colitis control treated with acetic acid rectally, C: UC treated with Turmeric (50 mg/kg), D: UC treated with Bromelain (100 mg/kg), E: UC treated with Combination of Bromelain (100 mg/kg) and Turmeric (50 mg/kg).

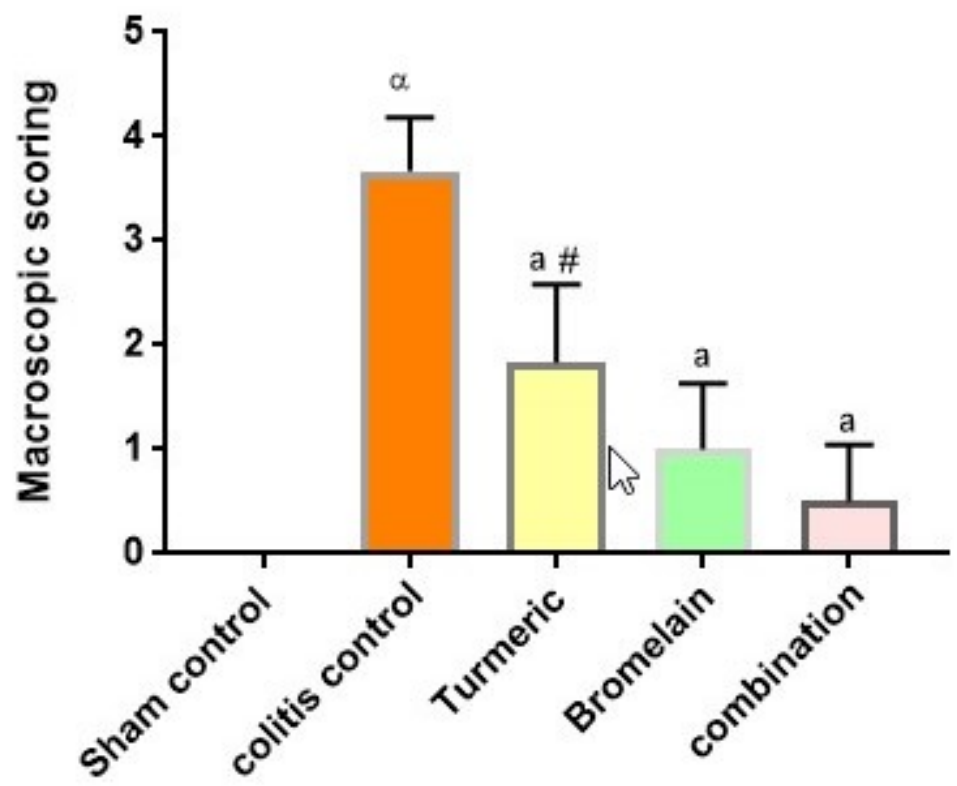

Groups

Figure 3: Effect of Bromelain and Turmeric on Macroscopic Scoring.

Results are expressed as Mean \pm S.E.M, P $<0.0001$ vs. Sham control. ${ }^{\text {ap }}<0.0001$ vs. Colitis control. ${ }^{\#} \mathrm{P}<0.002$ vs. Combination. 


\section{Effect of Bromelain and Turmeric on Colonic MPO}

Induction of colitis has significantly increased the colonic MPO levels in the colitis control group compared to the sham control group ( $\mathrm{P}<0.0001)$. Treatment with Turmeric and Bromelain alone has shown a significant decrease in MPO levels compared to the colitis control group ( $\mathrm{P}<0.0001)$. The combination of both turmeric and bromelain has shown a more significant reduction in MPO levels compared to monotherapies $(\mathrm{P}<0.002)$ [Figure 4].

\section{Effect of Bromelain and Turmeric on Histopathology}

Sham control (A) showed normal muscularis mucosa, the submucosa and, the mucosa with normal epithelial lining of the crypts, the goblet cells and, the lamina propria. Colitis control (B) showing the disruption of the epithelial mucosa layer of the villi with necrotic material in the lumen. Turmeric $(50 \mathrm{mg} / \mathrm{kg}$ ) (C) attenuated the extent and severity of the histological signs of cell damage.

Bromelain (100 mg/kg) (D) showed mild histological signs of cell damage. Combination (Turmeric $50 \mathrm{mg} / \mathrm{kg}$ and Bromelain $100 \mathrm{mg} / \mathrm{kg}$ ) (E) showed intact epithelial lining with normal muscularis mucosa, the submucosa and, the mucosa [Figure 5].

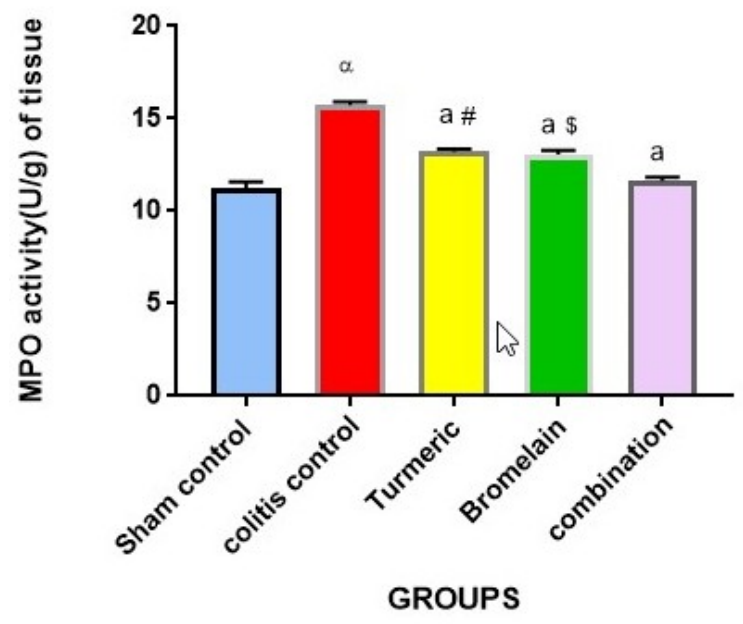

Figure 4: Effect of Bromelain and Turmeric on MPO levels

Results are expressed as Mean \pm S.E.M, $\square \mathrm{P}<0.0001$ vs. Sham control. ${ }^{a} \mathrm{P}<0.0001$ vs. Colitis control ${ }^{\#} \mathrm{P}<0.0001,{ }^{\$} \mathrm{P}<0.002$ vs. Combination.
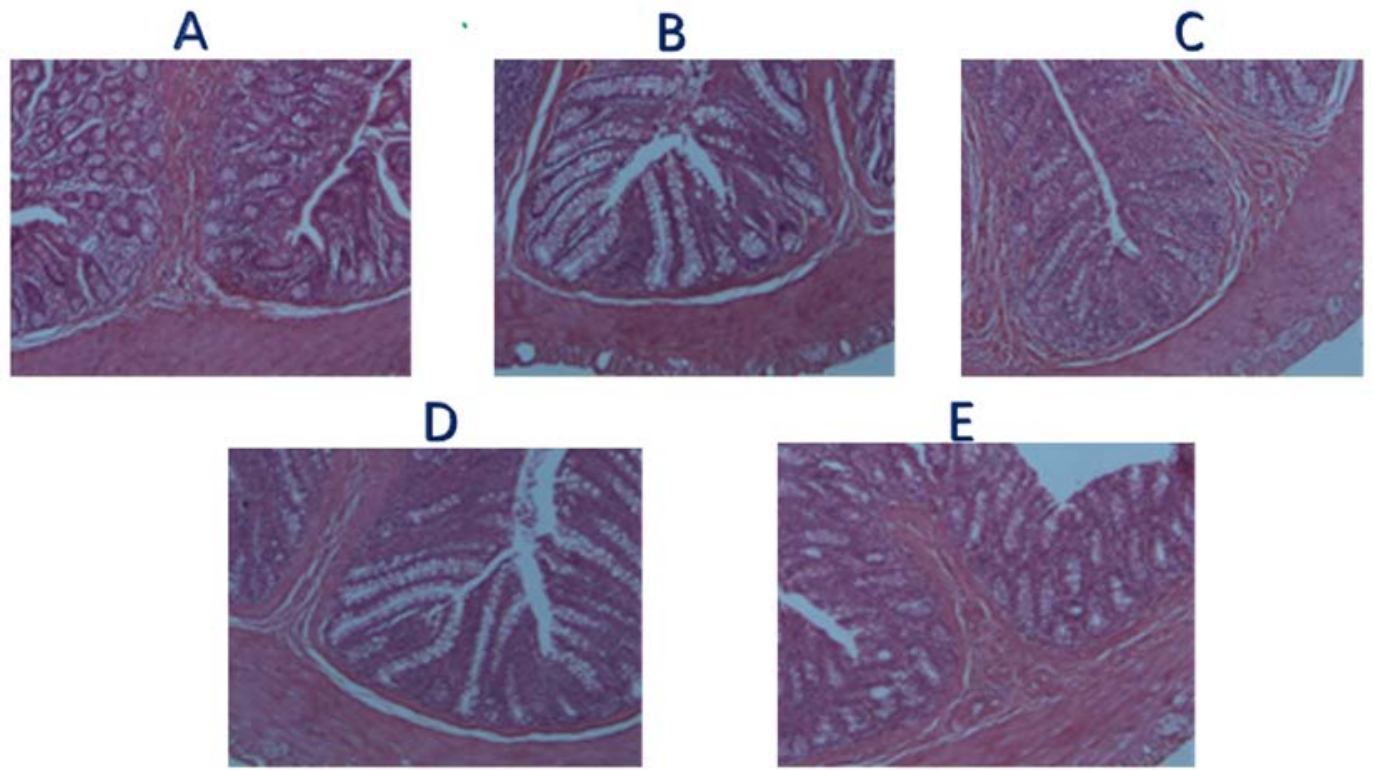

Figure 5: Showing $5 \mu$ thick Haemotoxylin \& Eosin stained section of the rat colonic tissue under 10X magnification. 
Inflammatory bowel disease is a chronic inflammatory disease of the gastrointestinal tract characterized by frequent diarrhea, abdominal pain, bleeding, anemia, weight loss and, chronic recurrent ulceration of the bowel. Causative agents are stress, diet, smoking and, microbial factors, which may lead to the development of carcinoma if untreated [16].

A recent study reported that "At the turn of the 21st century, inflammatory bowel disease has become a global disease with accelerating incidence in newly industrialized countries whose societies have become more westernized. Although the incidence is stabilizing in western countries, the burden remains high as prevalence surpasses $0 \cdot 3 \%$. These data highlight the need for research into the prevention of inflammatory bowel disease and innovations in health-care systems to manage this complex and costly disease” [17].

The main aim of the treatment of UC is to eliminate symptoms, prevent flare-ups (maintain long-term remission) and restore quality of life. The allopathic drugs presently available for the treatment of UC are used mainly to reduce infection and inflammation. They include aminosalicylates, antiinflammatory steroids, corticosteroids, immunosuppressants and, TNF (Tumour Necrosis Factor) inhibitors. However, side effects associated with these drugs are paramount and search is on for newer agents as well as for evidence based use of herbal medications [18].

Turmeric is a medicinal plant extensively used in Ayurveda, Unani and Siddha systems of medicine and is also used as a home remedy for various diseases. Curcumin, the main yellow bioactive component of turmeric has been found to have a broad spectrum of biological actions; anti-inflammatory, antioxidant, anticarcinogenic, Antimutagenic, anticoagulant, antibacterial, antifungal, antiulcer, etc. However, one of the major limitations with ingesting curcumin is its poor bioavailability, which appears to be primarily due to poor absorption, rapid metabolism, and rapid elimination. Several strategies have been investigated to improve curcumin’s bioavailability [19].

Bromelain is a complex natural mixture of proteolytic enzymes derived from pineapple (Ananas cosmosus) and found to exhibit several therapeutic properties, it is also used as a nutritional supplement to promote health. It is reported to enhance bioavailability and reduces the side effects that are associated with various antibiotics Furthermore, bromelain is reported to possess an immunomodulator, anti-metastatic, anti-edematous, anti-thrombotic and anti-inflammatory actions, etc [20,21].

Bromelain and Turmeric have been successfully used alone to reduce the swelling of the inflammation. Recent investigations have been proved that combined therapies are more effective than monotherapies. Moeinian $\mathrm{M}$ et al.,2014 have reported the priority of the combination of butyrate, L.casei and, L-carnitine in ameliorating the severity of colitis in comparison to monotherapies [22]. So the present study was planned to investigate the combined effect of bromelain and turmeric in acetic acid-induced Ulcerative colitis.

Acetic acid-induced ulcerative colitis has been found to have morphological similarities with the human disease of ulcerative colitis and also it is a standard reproducible model for UC, wherein inflammatory mediators such as reactive oxygen species, eicosanoids play a significant role. The underlying pathophysiological mechanisms involved in this include mucosal barrier destruction by chemical stimulation, enhanced vessel permeability, increased inflammatory mediators, etc [23].

Based on the clinical symptoms of UC like bloody stools, weight loss, Diarrhea, the scoring system is developed to assess the severity of the disease. In the present study colonic administration of $3 \% \mathrm{v} / \mathrm{v}$ acetic acid has shown induction of UC, as this significantly increased DAI score in the colitis control group compared to the sham control group. Upon treatment for 7 days with Turmeric \& Bromelain has significantly decreased DAI score compared to the colitis control group, also there is a significant decrease in DAI score in the combination group compared to individual therapies.

UC involves the activation of the Immune system and migration of activated neutrophils. Migration and accumulation of WBC \& Neutrophils to the inflamed intestinal mucosa is a prominent feature in ulcerative colitis [24]. This can be evaluated by measuring WBC \& neutrophil count. Induction of colitis has significantly increased WBC count in colitis control compared to the sham control group. Treatment with turmeric and bromelain alone has shown a significant decrease in WBC count compared to the colitis control group and a more significant decrease in WBC count was observed upon treating with the combination compared to monotherapies. 
A Significant increase in neutrophils count was observed in colitis control compared to the sham control group. Upon treatment with Turmeric and Bromelain alone and the combination has shown a significant decrease in neutrophils compared to the colitis control group, and no significant difference was observed in neutrophils count upon treating with the combination compared to monotherapies. Fitzhugh DJ et al demonstrated that bromelain can effectively decrease neutrophil migration to sites of acute inflammation [25].

The severity of the Ulcer was evaluated by a macroscopic scoring system [15]. Induction of colitis significantly increased ulcer score in colitis control compared to the sham control group. Treatment with Turmeric and Bromelain alone has shown a significant decrease in Ulcer Index compared to the colitis control group and upon treatment with the combination has shown a more significant reduction in the Ulcer score compared to monotherapies.

Myeloperoxidase (MPO), a component of monocyte and neutrophil granules, is used clinically as a marker of neutrophil infiltration into the intestinal mucosa. Curcumin has been shown to decrease intestinal inflammatory disease-associated MPO activity in animal models of colitis. In an immune-mediated model of mouse colitis, Mouzaoui et al. demonstrated that curcumin is capable of reducing neutrophil intestinal infiltration, thereby reducing MPO activity, as well as returning NO levels to baseline via inhibition of iNOS and reduced inflammatory cell infiltration [26]. In a rat model of TNBS-colitis, it has been investigated that treatment with curcumin significantly reduced the activity of MPO. Similarly, in a rat methotrexate-colitis model, curcumin decreased intestinal MPO and increased levels of free radical-scavenging superoxide dismutase $[27,28]$.

Myeloperoxidase is an hemoenzyme, which is released from neutrophils to eradicate pathogens. Therefore, activated neutrophils may contribute to tissue damage at sites of inflammation. Induction of colitis has significantly increased the colonic MPO levels in colitis control compared to the sham control group. Treatment with Turmeric and Bromelain alone and combination groups have shown a significant decrease in MPO levels compared to the colitis control group. However, no significant difference was observed in MPO levels of the combination compared to monotherapies.
In ulcerative colitis, mucosal healing may remain the ultimate therapeutic goal, because the disease is limited to the mucosa. The progression of inflammation in UC is associated with several mucosal changes, vascular congestion, erythema, and granularity. As the inflammation becomes more severe, spontaneous bleeding, erosions and, ulcers develop. An International Organization of Inflammatory Bowel Disease (IOIBD) task force defined mucosal healing in UC as the absence of friability, blood, erosions, and ulcers in all sections of the colonic mucosa [29].

In Histological findings such as mucosal edema, disruption of the epithelial mucosal layer of the villi and necrosis of the colon is considered a reliable sensitivity indicator of the severity and extent of inflammatory response [1]. Sham control showed normal muscularis mucosa, the submucosa and, the mucosa with normal epithelial lining of the crypts, the goblet cells and, the lamina propria. Whereas, Colitis control (acetic acid-induced) showing the disruption of the epithelial mucosa layer of the villi with necrotic material in the lumen and Turmeric (50 mg/kg) group attenuated the extent and severity of the histological signs of cell damage, Bromelain(100 mg/kg) showed mild histological signs of cell damage and Combination - (Turmeric $50 \mathrm{mg} / \mathrm{kg}$ and Bromelain $100 \mathrm{mg} / \mathrm{kg}$ ) showed intact epithelial lining with normal musularis mucosa, the submucosa and, the mucosa.

\section{CONCLUSION}

The findings of the present study clearly show that the combination of Bromelain and Turmeric has significantly suppressed the DAI score by increasing body weight and decreasing stool consistency, rectal bleeding compared to individual therapies. The combination of Turmeric and Bromelain has shown a significant reduction in macroscopic scoring, ulcer index compared to individual therapies. A significant change was observed in WBC, DLC Count and, MPO levels in individual and combination groups compared to the colitis group but no significant difference was observed in the case of the combination compared to monotherapies. While both of these herbs complement each other and they have been successfully used alone to reduce the inflammation in UC, and so on combination therapy has proved more effective compared to monotherapies, the present study suggests that combined action of bromelain and turmeric is more effective in reducing the inflammation in ulcerative colitis. Further studies are 
required to investigate the mechanism involved in the synergistic effect of Bromelain and Turmeric Combination.

\section{FINANCIAL ASSISTANCE \\ Nil}

\section{CONFLICT OF INTEREST}

The authors declare no conflict of interest.

\section{AUTHOR CONTRIBUTION}

A. Lalitha Devi, Y. Sree Hari and, Abdul Samad designed the work and made necessary corrections and, revisions in the manuscript. K. B. Keerthana collected the content and performed the literature review and also contributed to writing the manuscript. All the authors drafted the final manuscript. R. Padmavathi supervised the work and involved in designing and conducting experimental work, preparation of manuscript

\section{REFERENCES}

[1] Langner C, Magro F, Driessen A, Ensari A, Mantzaris GJ, Villanacci V, Becheanu G, Borralho Nunes P, Cathomas G, Fries W, Jouret-Mourin A, Mescoli C, De Petris G, Rubio CA, Shepherd NA, Vieth M, Eliakim R, Geboes K. The histopathological approach to inflammatory bowel disease: A practice guide, Springer Verlag, 2014.

[2] Wilks S, Moxon W. Lectures on Pathological Anatomy. LANE Libr., 733 (1875).

[3] Crohn BB, Ginzburg L, Oppenheimer GD. Regional ileitis: A pathologic and clinical entity. J. Am. Med. Assoc., 99, 1323-9 (1932).

[4] Liu TC, Stappenbeck TS. Genetics and Pathogenesis of Inflammatory Bowel Disease. Annu. Rev. Pathol. Mech. Dis., 11, 127-48 (2016).

[5] Solanki R, Madat D, Chauhan K, Parmar L. Recent approaches in pathogenesis of inflammatory bowel disease, 2010.

[6] Su HJ, Chiu YT, Chiu CT, Lin YC, Wang CY, Hsieh JY, Wei SC. Inflammatory bowel disease and its treatment in 2018: Global and Taiwanese status updates. J. Formos. Med. Assoc., 118, 1083-92 (2019).

[7] Zhang, Yi-Zhen, and Yong-Yu Li. Inflammatory bowel disease: pathogenesis. World journal of gastroenterology: WJG 20, no. 1,91 (2014).

[8] Baliga MS, Joseph N, Venkataranganna M V., Saxena A, Ponemone V, Fayad R. Curcumin, an active component of turmeric in the prevention and treatment of ulcerative colitis: Preclinical and clinical observations. Food Funct., 3, 1109-17 (2012).

[9] Ma Z, Wang N, He H, Tang X. Pharmaceutical strategies of improving oral systemic bioavailability of curcumin for clinical application. J. Control. Release, 316, 359-80 (2019).

[10] Chakraborty M, Bhattacharjee A, Kamath JV. Cardioprotective effect of curcumin and piperine combination against cyclophosphamide-induced cardiotoxicity. Indian J. Pharmacol., 49, 65-70 (2017).

[11] Hale LP, Greer PK, Trinh CT, Gottfried MR. Treatment with oral bromelain decreases colonic inflammation in the IL-10-deficient murine model of inflammatory bowel disease. Clin. Immunol., 116, 135-42 (2005).

[12] Zhou Z, Wang L, Feng P, Yin L, Wang C, Zhi S, Dong J, Wang J, Lin Y, Chen D, Xiong Y, Peng J. Inhibition of epithelial TNF- $\alpha$ receptors by purified fruit bromelain ameliorates intestinal inflammation and barrier dysfunction in colitis. Front. Immunol., 8, (2017).

[13] Thippeswamy BS, Mahendran S, Biradar MI, Raj P, Srivastava K, Badami S, Veerapur VP. Protective effect of embelin against acetic acid induced ulcerative colitis in rats. Eur. J. Pharmacol., 654, 100-5 (2011).

[14] Kottke-Marchant K, Davis BH. Laboratory Hematology Practice. Wiley-Blackwell, (2012).

[15] Patil NR, Rasal VP, Malabade RH. Screening of mandarin oil on indomethcin induced inflammatory bowel disease in wistar rats. Indian J. Pharm. Educ. Res., 48, 1-6 (2014).

[16] Guan Q. A Comprehensive Review and Update on the Pathogenesis of Inflammatory Bowel Disease. J. Immunol. Res., 2019, (2019).

[17] Ng SC, Shi HY, Hamidi N, Underwood FE, Tang W, Benchimol EI, Panaccione R, Ghosh S, Wu JCY, Chan FKL, Sung JJY, Kaplan GG. Worldwide incidence and prevalence of inflammatory bowel disease in the 21st century: a systematic review of population-based studies. Lancet, 390, 2769-78 (2017).

[18] Tabassum N, Hamdani M, Hussain Najar I. BRITISH BIOMEDICAL BULLETIN Original Natural Treatment for Inflammatory Bowel Disease, 2013.

[19] Hewlings S, Kalman D. Curcumin: A Review of Its Effects on Human Health. Foods, 6, 92 (2017).

[20] Pavan R, Jain S, Shraddha, Kumar A. Properties and Therapeutic Application of Bromelain: A Review. Biotechnol. Res. Int., 2012, 1-6 (2012). 
[21] Rathnavelu V, Alitheen NB, Sohila S, Kanagesan S, Ramesh R. Potential role of bromelain in clinical and therapeutic applications (Review). Biomed. Reports, 5, 283-8 (2016).

[22] Moeinian M, Ghasemi-Niri SF, Mozaffari S, Abdolghaffari AH, Baeeri M, Navaea-Nigjeh M, Abdollahi M. Beneficial effect of butyrate, Lactobacillus casei and L-carnitine combination in preference to each in experimental colitis. World J. Gastroenterol., 20, 10876-85 (2014).

[23] Fabia R, Willen R, Arrajab A, Andersson R, Ahren B, Bengmark S. Acetic acid-induced colitis in the rat: A reproducible experimental model for acute ulcerative colitis. Eur. Surg. Res., 24, 211-25 (1992).

[24] Sartor RB. Mechanisms of disease: Pathogenesis of Crohn's disease and ulcerative colitis. Nat. Clin. Pract. Gastroenterol. Hepatol., 3, 390-407 (2006).

[25] Fitzhugh DJ, Shan S, Dewhirst MW, Hale LP. Bromelain treatment decreases neutrophil migration to sites of inflammation. Clin. Immunol., 128, 66-74 (2008).
[26] Mouzaoui S, Rahim I, Djerdjouri B. Aminoguanidine and curcumin attenuated tumor necrosis factor (TNF)- $\alpha$-induced oxidative stress, colitis and hepatotoxicity in mice. Int. Immunopharmacol., 12, 302-11 (2012).

[27] Jiang H, Deng CS, Zhang M, Xia J. Curcumin-attenuated trinitrobenzene sulphonic acid induces chronic colitis by inhibiting expression of cyclooxygenase-2. World $J$. Gastroenterol., 12, 3848-53 (2006).

[28] Song WB, Wang YY, Meng FS, Zhang QH, Zeng JY, Xiao LP, Yu XP, Peng D dan, Su L, Xiao B, Zhang ZS. Curcumin protects intestinal mucosal barrier function of rat enteritis via activation of MKP-1 and attenuation of p38 and NF-кB activation. PLoS One, 5, (2010).

[29] Walsh A, Palmer R, Travis S. Mucosal healing as a target of therapy for colonic inflammatory bowel disease and methods to score disease activity. Gastrointest. Endosc. Clin. N. Am., 24, 367-78 (2014). 